\title{
Pendulum-type laser strainmeter
}

\author{
Grigory Ivanovich Dolgikh
}

Received: 29 April 2015/Accepted: 7 July 2015/Published online: 2 September 2015

(C) The Author(s) 2015. This article is published with open access at Springerlink.com

\begin{abstract}
Constructive disposition principles of the basic optical units of Michelson interferometers are used in making one-coordinate laser strainmeters of unequal-arm type. The fundamental advantages of pendulum-type laser strainmeter more than classical-type laser strainmeter are displayed.
\end{abstract}

Keywords Amplitude-frequency characteristic . Classical-type laser strainmeter · Elastic wave . Infrasound · Pendulum-type laser strainmeter

\section{Introduction}

Currently, the laser strainmeters designed for recording the gravity waves, studying the geodynamical and geophysical processes (Park et al. 2008; Garoi et al. 2008; Jahr et al. 2006), examining effects of all kinds of hydrospheric and atmospheric processes on crustal microdeformations and its seismicity (Takemoto et al. 2004; Dolgikh and Ovcharenko 2008; Dolgikh et al. 2002, 2004, 2007) are successfully on operation. The existing laser strainmeters can be divided into three types: (1) one-coordinate, (2) two-coordinate, and (3) three-coordinate. The optical schemes of all laser strainmeters are based on Michelson interferometers (in the main) and Fabry-Perot interferometers. As the light sources in them, the lasers with the highly stable frequency (wave length) are used. The one-coordinate, two-coordinate, and

G. I. Dolgikh $(\bowtie)$

V.I. Il'ichev Pacific Oceanological Institute of the Far Eastern Branch, Russian Academy of Sciences, Vladivostok, Russia 690041

e-mail: novikova@poi.dvo.ru three-coordinate laser strainmeters are produced respectively based on the equal-arm or unequal-arm principles. In the paper, we will study features of the one-coordinate laser strainmeters based on the unequal-arm principle. In this case, the optical elements of the one-coordinate laser strainmeter are mounted to two blocks which are rigidly connected by the terrestrial rocks (Takemoto et al. 2004). The measuring arm of the laser strainmeter is connected between two blocks while the reference arm is fixed on one of the blocks. Such a laser strainmeter is designed for highprecision measurements of change in distance between the above-mentioned two blocks. Technical characteristics of the laser strainmeters depend mainly on the frequency stability of lasers used $(\Delta L / L=|-\Delta f / f|$, where $\Delta L$ is the base displacement of the strainmeter actuator arm, the length of which is $L, \Delta f / f$ is the frequency stability of laser), ways of mounting the strainmeter blocks and basic parameters of their environment.

An application of lasers with the long-term stability of $10^{-12} / 10^{-13}$ allows measurements of strain variations on the base of a device (strainmeter actuator arm) with an accuracy of $10^{-12} / 10^{-13}$. In the following sections of the paper, we will consider the peculiarities of the amplitudefrequency characteristics of the classical-type laser strainmeters in which the optical elements of the one-coordinate laser strainmeter are mounted on two blocks which are rigidly connected with the terrestrial rocks and pendulumtype laser strainmeter in which angel reflector is mounted to the pendulum system. The pendulum-type laser strainmeter can be regarded as a hybrid of the interferometer with free masses and interferometer with rigidly fixed mirrors. In the early time, the pendulum systems were used in the interferometers in order to reduce the effect of the geophysical noise on mirrors [see, for example, Abbott 
et al. (2009), Acernese et al. (2015) and Akutsu (2015)]. The comparison study are also made between the optical and electronic strainmeters (McDonnell et al. 2008). In our study, the pendulum system is used for improving the amplitude-frequency characteristics of laser strainmeters when recording the geophysical signals in the high-frequency domain. Below we will report some experimental results obtained by use of the classical- and pendulum-type laser strainmeters respective when recording the geophysical processes in the infrasonic range and signals of the low-frequency underwater acoustic projector.

\section{Classical-type laser strainmeter}

On the left side of Fig. 1, one-coordinate laser strainmeter based on block-designed with unequal-arm is presented. Both angle reflectors are mounted on two blocks rigidly connected with elastic medium (Earth's crust). A change in distance between the two blocks is measured using the interference techniques. Let's call such a laser strainmeter the one-coordinate classical-type laser strainmeter. A change in distance between the blocks can be caused by different processes including the wave oscillatory, meteorological, geophysical, geodynamic factors etc. When arranging the laser strainmeter on the Earth's surface, it can record the surface Rayleigh waves, longitudinal and transversal waves. For the purposes of investigating the features of amplitude-frequency characteristics of the classical-type laser strainmeter, we take the simplest harmonic longitudinal wave as the example. And the two blocks of laser strainmeter are arranged on the uniform medium. When longitudinal wave propagates through the laser strainmeter axis, the displacement of the one point $x_{1}$ in crust can be described by the below equation:

$u_{1}=A_{0} \cos \left(\omega t-k x_{1}\right)$,

where $u_{1}$ is displacement of the Earth's crust particle at point $x_{1}, A_{0}$ is the harmonic wave amplitude, $k=2 \pi / \lambda$ is the wave number, $\lambda$ is the wavelength, $\omega=2 \pi v$ is the cyclic frequency, $v$ is the wave frequency and $t$ is the time. And the classical-type laser strainmeter, will also record the displacement which equal to distance change between the blocks

$\Delta L=u_{2}-u_{1}=2 A_{0} \sin \left(\frac{k L}{2}\right) \sin \left(\omega t-k x_{1}-\frac{k L}{2}\right)$,

where $L$ is the length of the strainmeter actuating arm (distance between the blocks of a laser strainmeter), $x_{1}$ is the coordinate of the first block of strainmeter, $x_{2}$ is the coordinate of the second block of strainmeter, $x_{2}=x_{1}+L$, $u_{2}=A_{0} \cos \left(\omega t-k x_{2}\right)$, is the displacement of the Earth's crust particle at point $x_{2}$. As seen from Eq. (2), the wave amplitude $A_{\text {rec }}$ recorded by the laser strainmeter depends on $A_{0}, L$ and $\lambda$.

When longitudinal harmonic wave in the frequency of $0-30 \mathrm{~Hz}$ propagates, change of amplitude with the frequency by the one-coordinate laser strainmeter are recorded. Let us assume that the length of the laser strainmeter actuator arm is $1000 \mathrm{~m}$, wave amplitude is 1 and its

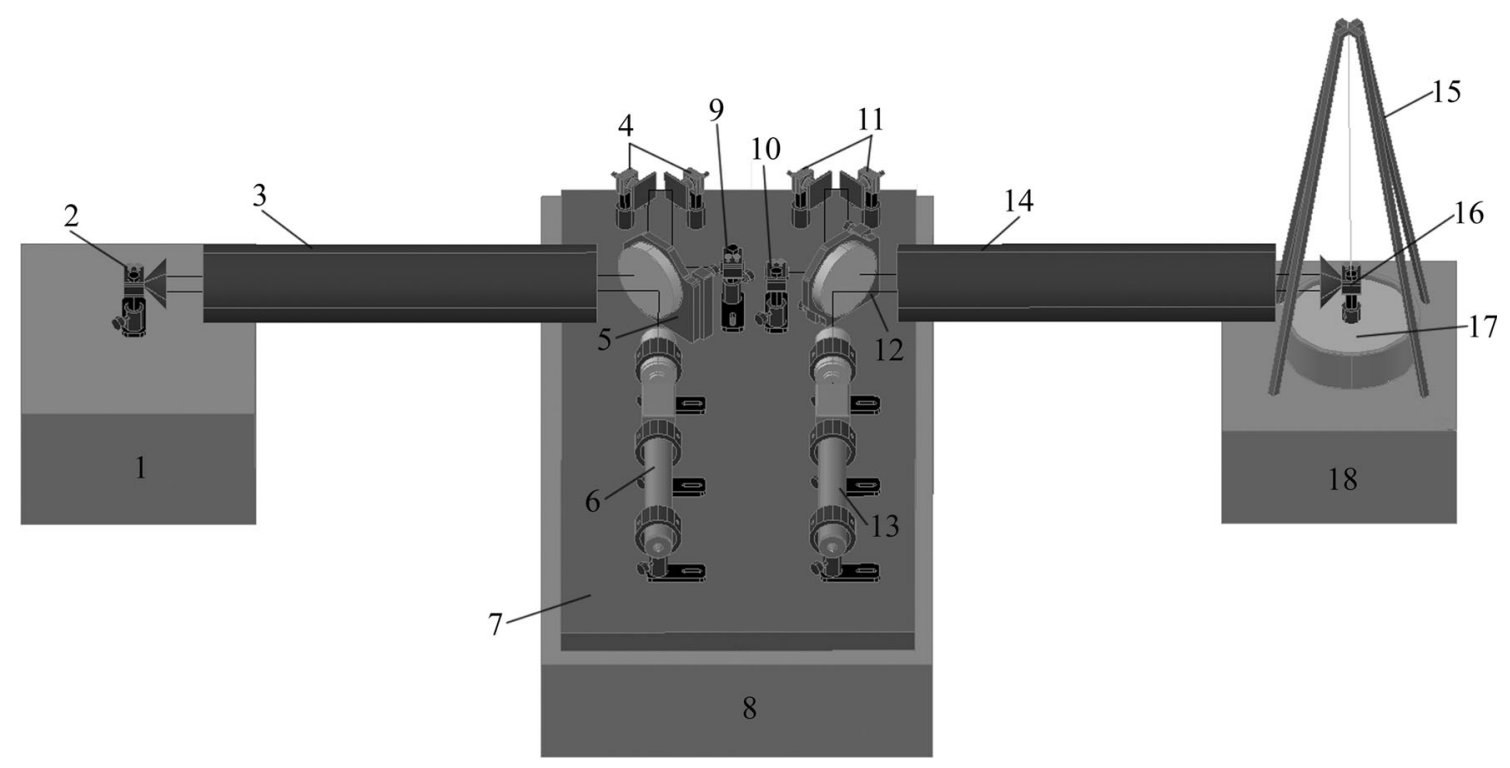

Fig. 1 Block-scheme of the one-coordinate laser strainmeters of unequal-arm type: classical-type laser strainmeter and pendulum-type laser strainmeter $(1,8,18$ rocks of the Earth crust; 7 block of the central part of Michelson interferometers; 2, 16 block of the angel reflector; 3, 14 optical beam waveguide; 6,13 frequency-stabilized laser, optical gate, collimator; 5, 12 dividing plate; 4, 11 reflecting mirrors on piezoceramic cylinders; 9 , 10 photodetector, 15 pendulum, 17 cuvette with oil) 

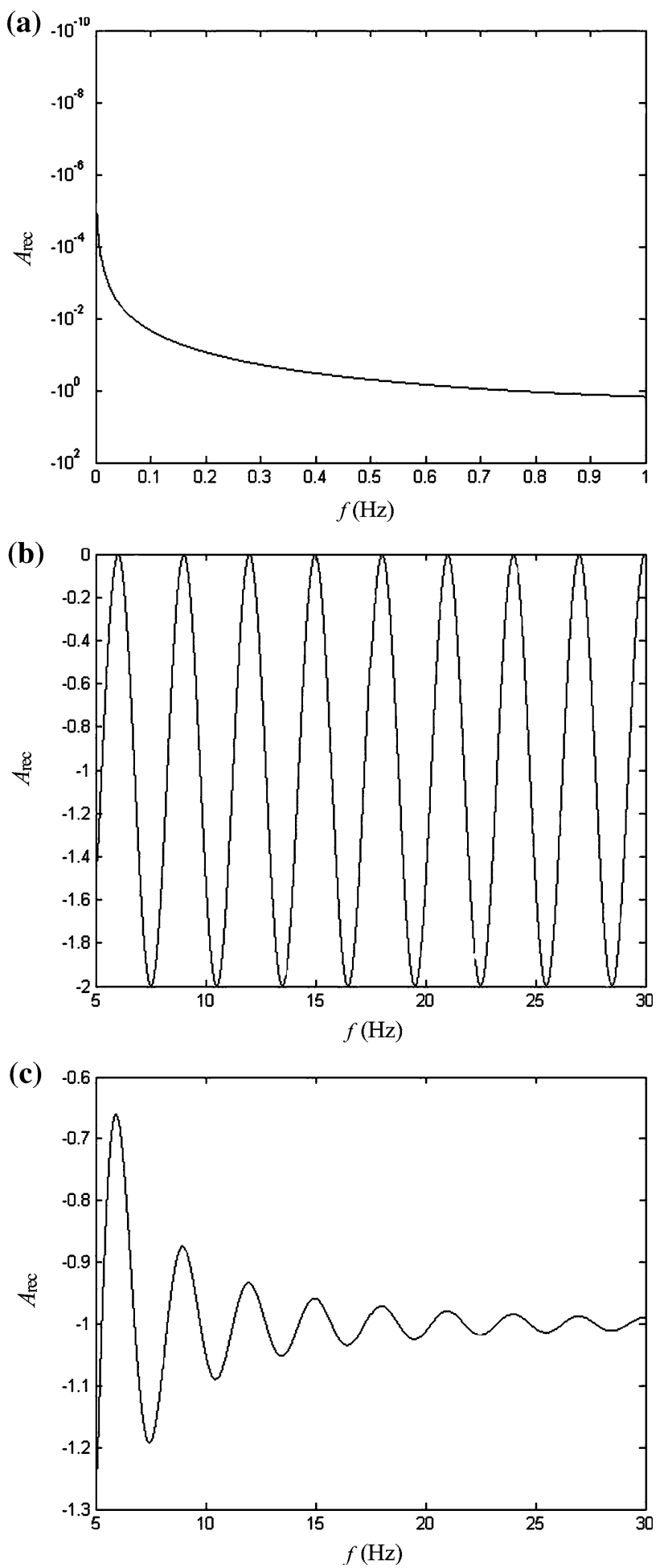

Fig. 2 Amplitude-frequency relation of the $1000 \mathrm{~m}$ long actuator arm one-coordinate classical-type laser strainmeter in the frequency range of $0-1 \mathrm{~Hz}$ (a) and in the frequency range of 5 to $30 \mathrm{~Hz}(\mathbf{b})$. Amplitude-frequency relation of the $1000 \mathrm{~m}$ one-coordinate pendulum-type laser strainmeter in the frequency range of 5 to $30 \mathrm{~Hz}(\mathbf{c})$ velocity is equal to $3000 \mathrm{~m} / \mathrm{s}$. The amplitude-frequency characteristic of the one-coordinate classical-type laser strainmeter with a length of actuating arm of $1000 \mathrm{~m}$ within the infrasonic range $(0-1 \mathrm{~Hz})$ is shown in Fig. $2 \mathrm{a}$ where frequency in $\mathrm{Hz}$ is plotted on the abscissa while $A_{\text {rec }}$ on the ordinate. In the high-frequency band, beginning from frequency of about $5 \mathrm{~Hz}$, the amplitude-frequency characteristic of this laser strainmeter varies harmonically (beat zone) (Fig. 2b). In other words, the instrument produces in some frequencies double wave amplitude (in modulus, $\left|A_{\text {rec }}\right|=2 A_{0}$ ) while in other ones 0 instead of real amplitude equal to 1 . That is, it is difficult to record absolute amplitude correctly in the beat zone due to periodic variations of the amplitude-frequency characteristic. It is impossible to calculate theoretically and determine experimentally all of specific features of the particular laser strainmeters due to inhomogeneous structure of the Earth crust within a zone of its arrangement. Therefore, the laser strainmeter with a length of the actuating arm of $1000 \mathrm{~m}$ can be successfully applied for recording the displacements in the infrasonic range while it is unsuitable for recording the oscillations of waves in order of $1 \mathrm{~Hz}$ and higher. Can these beats be eliminated? Below, we will consider a basic procedure which allows not only to eliminate the beats but also to measure the absolute amplitudes of elastic waves at frequencies higher than $1 \mathrm{~Hz}$.

\section{Pendulum-type laser strainmeter}

As shown in the right side of the Fig. 1, both blocks are on the elastic medium, one optical element is arranged on the middle block as the same as left side, the other one is connected with pendulum system having the resonance frequency $v_{0}$ and quality factor $Q$. For the middle block, the angle reflector is only mounted. The other block is hung on the inextensible thread possessing a negligible mass as compared with total mass of the block and angle reflector. The length of the thread is much greater than geometrical sizes of the block and angle reflector. In this case, the block with angle reflector can be, within a certain approximation, taken as a point mass. Thus, we can say that this is a mathematical pendulum. We call a laser strainmeter designed under this principle as the pendulum-type laser strainmeter. When a harmonic longitudinal wave propagates through the pendulum-type laser strainmeter, the differential equation of motion of induced oscillations can be written as follows $\Delta L=u_{2}-u_{1}$ :

$\ddot{x}+2 \gamma \dot{x}+\omega_{0}^{2} x=f_{0} \cos \omega t$, 
where $\gamma=\omega_{0} / 2 Q, \omega_{0}=2 \pi v_{0}, v_{0}$ is natural frequency, $f_{0} \cos \omega t$ is driving force.

Solving this equation at $f_{0}=F_{0} / m=A_{0} \omega_{0}^{2}$, the displacement $u_{2}$ of the angle reflector (pendulum) at one point with coordinates $x_{2}$ :

$$
\begin{aligned}
u_{2}= & A_{0}\left\{\frac{\omega_{0}^{2}}{\sqrt{\left(\omega_{0}^{2}-\omega^{2}\right)^{2}+4 \gamma^{2} \omega^{2}}}\right. \\
& \left.\cos \left[k x_{1}+k L-\omega t+\arctan \left(\frac{2 \gamma \omega}{\omega_{0}^{2}-\omega^{2}}\right)\right]\right\} .
\end{aligned}
$$

A displacement of the block rigidly mounted on the elastic medium at the point $x_{1}$ will be described by the following equation:

$u_{1}=A_{0} \cos \left(k x_{1}-\omega t\right)$.

Therefore, in case of propagation of the harmonic longitudinal wave along the pendulum-type laser strainmeter axis, the strainmeter will record a change in its base which can be written as follows:

$$
\begin{aligned}
\Delta L= & A_{0}\left\{\frac{\omega_{0}^{2}}{\sqrt{\left(\omega_{0}^{2}-\omega^{2}\right)^{2}+4 \gamma^{2} \omega^{2}}}\right. \\
& \times \cos \left[k x_{1}+k L-\omega t+\arctan \left(\frac{2 \gamma \omega}{\omega_{0}^{2}-\omega^{2}}\right)\right] \\
& \left.-\cos \left(k x_{1}-\omega t\right)\right\} .
\end{aligned}
$$

In order to obtain the numerical estimates, we will consider a recording of the harmonic longitudinal wave by the one-coordinate laser strainmeter of pendulum type. Let us assume that the length of actuating arm (distance between the blocks) is $1000 \mathrm{~m}$, velocity of the harmonic longitudinal wave is $3000 \mathrm{~m} / \mathrm{s}$ and wave amplitude is 1 . Let the $Q$-factor of the pendulum system be 100 while its resonance frequency $3 \mathrm{~Hz}$. In the infrasonic range $(0-1 \mathrm{~Hz})$, the amplitude-frequency characteristic of the one-coordinate laser strainmeter of pendulum type is practically identical to that for one-coordinate laser strainmeter of classical type. When increasing the measuring arm of the pendulum-type laser strainmeter, its sensitivity within infrasonic range enhances also. In the high-frequency spectral range, the amplitude-frequency characteristics of the pendulum-type laser strainmeter (See Fig. 2c) are distinctly different from those of the classicaltype laser strainmeter. With increasing the frequency, the amplitude of beats slowly decreases and nears to 1 in absolute value, i.e., the laser strainmeter of the pendulum type can record the true amplitude of the transmitted harmonic wave of longitudinal type at high frequencies. In the frequency range of $1-5 \mathrm{~Hz}$, the amplitude-frequency characteristic of the classical-type laser strainmeter beats also while that of the pendulum-type laser strainmeter at a frequency of $3 \mathrm{~Hz}$ presents resonance phenomenon. The amplitude value at resonance depends on the $Q$-factor of a pendulum. So, at the pendulum $Q$-factor of 100 , resonance frequency of $0.33 \mathrm{~Hz}$ and wave amplitude equal to 1 , the resonance amplitude will be about 100 . Therefore, the resonance amplitude will become more and more with the increase of $Q$-factor.

Comparison of recording of longitudinal wave with time by the laser strainmeters of pendulum and that of classical types in the frequency range of $0-1 \mathrm{~Hz}$ and $5-30 \mathrm{~Hz}$ are made, respectively. $x_{1}$ is taken as origin of coordinates, i.e., $x_{1}=0$.

In Fig. 3a, a change with time of displacement in the elastic medium caused by the propagating elastic wave is shown in the frequencies range of $0-1 \mathrm{~Hz}$. In Fig. 3b, c, a change of displacement with time in the elastic medium (at $x_{1}=0$ ) is presented when recording of elastic wave by laser strainmeters of classical and pendulum types, respectively. A comparison of these figures allows drawing the following conclusions: (1) in the frequency range of $0-1 \mathrm{~Hz}$, the laser strainmeters produce practically the same result; (2) in this frequency range, both strainmeters demonstrate basically the same character of the displacement variations in the elastic medium caused by the propagating wave.

In Fig. 4a, the time variation of displacement in the elastic medium ( $x_{1}=0$, origin of coordinates) when elastic wave propagates through with the frequency range of $5-30 \mathrm{~Hz}$ is presented. Fig. $4 \mathrm{~b}$, c give a time change of the displacement in the elastic medium (at $x_{1}=0$ ) recorded by the classical-type and pendulum-type laser strainmeters, respectively. A comparison of these figures allows draw the following conclusions: (1) in the frequency range of $5-30 \mathrm{~Hz}$ and higher, the pendulum-type laser strainmeter can be record accurately the displacement variations in the elastic medium (with regard to multiplication by -1 and light phase shift) caused by the propagating wave; (2) whereas classical-type laser strainmeter can not do that well.

\section{Case study: Comparison of recording by classical- type and pendulum-type}

The layout diagram of laser strainmeters of pendulum and classical types presented in Fig. 1 was implemented into practice. The devices were installed on Shults cape (southern Primorsky Krai of Russia) at a depth of 3-5 m below the surface. The length of actuating arms of the laser strainmeters is $52.5 \mathrm{~m}$. Central interference blocks of laser strainmeters are situated on one block which is rigidly 

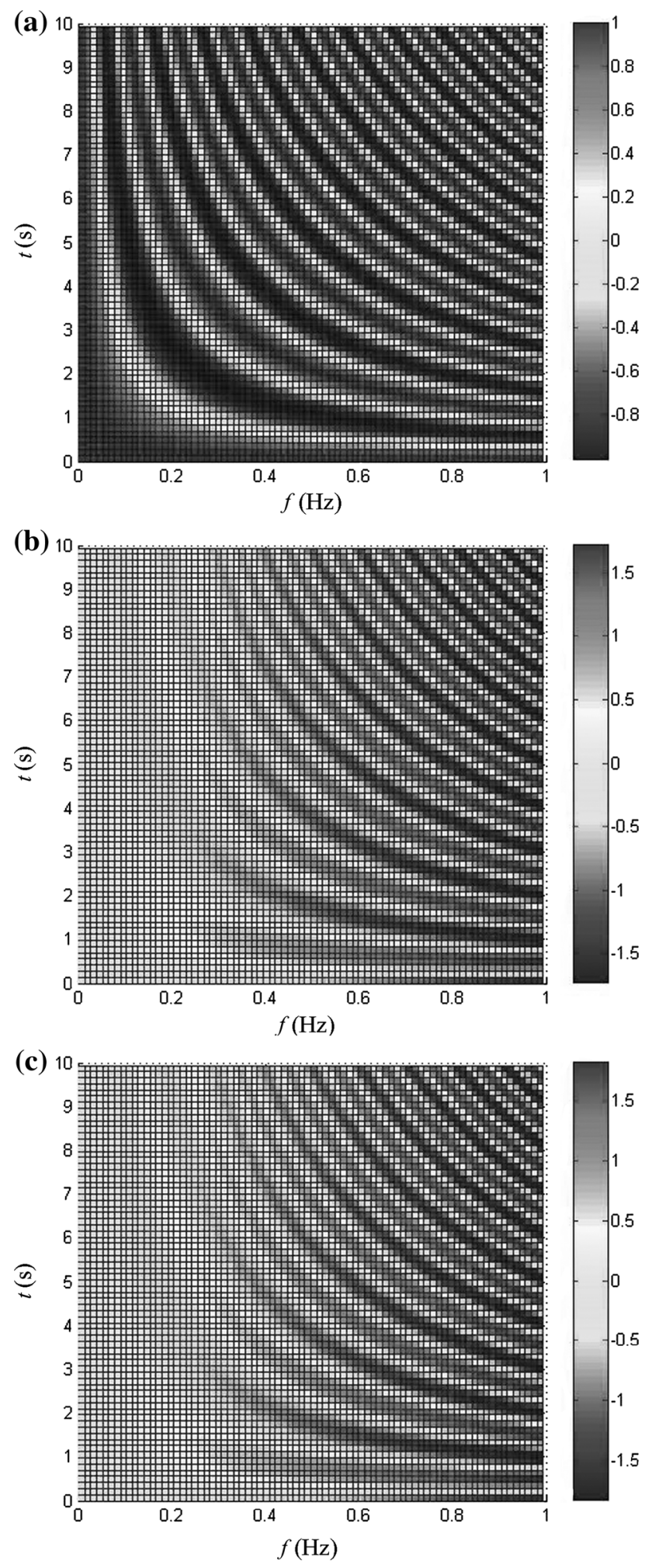

Fig. 3 Time change of the displacement recordings in the elastic medium when elastic wave propagates through (a), time change of the displacement recordings in the elastic medium by a classical-type laser strainmeter with actuating arm of $1000 \mathrm{~m}(\mathbf{b})$, time change of the displacement recordings in the elastic medium by a pendulum-type laser strainmeter with actuating arm of $1000 \mathrm{~m}(\mathbf{c})$
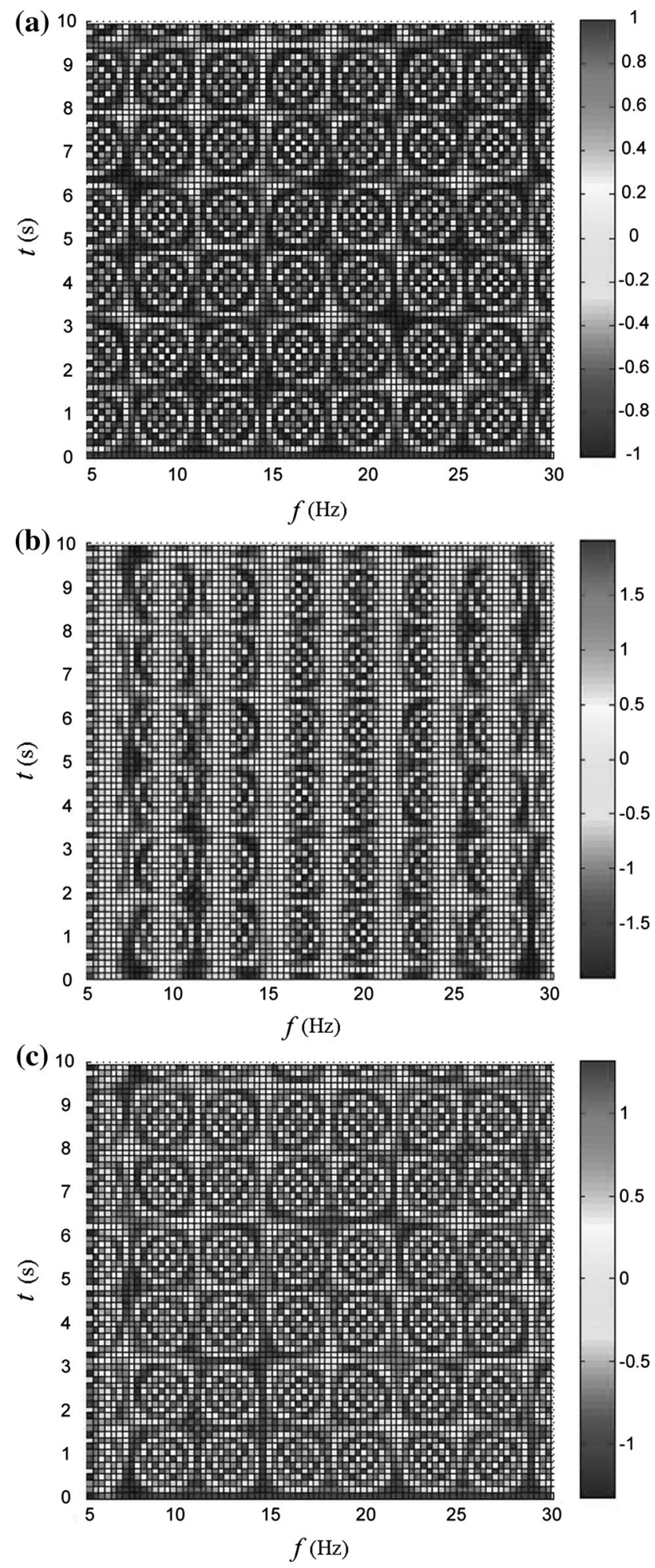

Fig. 4 Time change of the displacement recordings in the elastic medium when elastic wave propagates through (a), time change of the displacement recordings in the elastic medium by a classical-type laser strainmeter with actuating arm of $1000 \mathrm{~m}(\mathbf{b})$, time change of the displacement recordings in the elastic medium by a pendulum-type laser strainmeter with actuating arm of $1000 \mathrm{~m}$ (c) 
mounted on the crustal rocks. The angle reflector of the classical-type laser strainmeter is fixed on the block which is connected with the granite rock. The angle reflector of the pendulum-type laser strainmeter is connected with the pendulum system, the oscillation period of which is $3 \mathrm{~s}$. The pendulum is partially damped out due to immersion of its blades into oil. As the light source in the interferometers, the frequency-stabilized helium-neon lasers having a long-term stability up to eleventh decimal are used. The low-frequency underwater acoustic radiators with given radiation frequency is taken as the artificial sources, which is operated at sea $19 \mathrm{~km}$ apart from the Shultz cape to the south. The measurement axes of laser strainmeters are oriented along one straight line which is at angle of $18^{\circ}$ to NS. Since last year, the synchronous measurements of variations of Earth's crustal deformations caused by different geosphere processes are performed. In the infrasonic spectral range, the records of both laser strainmeters are practically identical. But in the high-frequency range of spectrum (more than $5 \mathrm{~Hz}$ ), we should compare the recording sensitivities of the two laser strainmeters when to the particular artificial sources. Fig. 5 gives the spectra recordings of laser strainmeters of classical (Fig. 5a) and pendulum (Fig. 5b) types, respectively. It can be seen from Fig. 5 that the amplitude recorded by pendulum-type laser
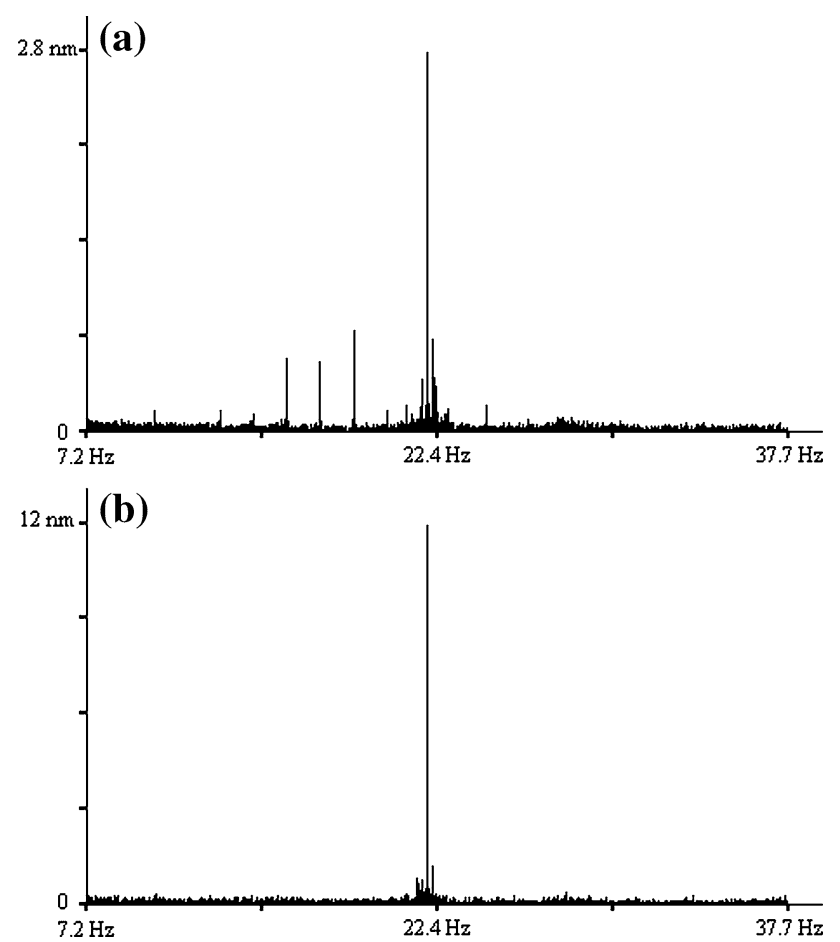

Fig. 5 The amplitude spectra recordings by classical-type laser strainmeter (a) and pendulum-type (b) of a low-frequency hydroacoustic radiator with radiation frequency of $22 \mathrm{~Hz}$ strainmeters is much greater than that by classical-type under the frequency of acoustic radiator $(22 \mathrm{~Hz})$, which can demonstrate that the sensitivity of the pendulum-type laser strainmeter is certainly much higher than that of classicaltype for this frequency range.

\section{Conclusions}

Based on the above-mentioned study, some conclusions can be drawn:

In the frequency of infrasonic $(0-1 \mathrm{~Hz})$, both classicaltype and pendulum-type laser strainmeters can record longitudinal wave well; while in the frequency range equal to and larger than $5 \mathrm{~Hz}$, the sensitivity of pendulum-type laser strainmeter is superior to classical-type. All these demonstrate that pendulum-type laser strainmeter can record well microdisplacements of its environment caused by the elastic waves propagating whereas the classical-type laser strainmeter is not capable of recording these variations in this frequency range.

Acknowledgments The work was supported financially in part by the Russian Science Foundation (RSF) (Grant No. 14-17-00041).

Open Access This article is distributed under the terms of the Creative Commons Attribution 4.0 International License (http://crea tivecommons.org/licenses/by/4.0/), which permits unrestricted use, distribution, and reproduction in any medium, provided you give appropriate credit to the original author(s) and the source, provide a link to the Creative Commons license, and indicate if changes were made.

\section{References}

Abbott BP, Abbott R, Adhikari R et al (2009) LIGO: the laser interferometer gravitational-wave observatory. The LIGO scientific collaboration. Rep Prog Phys 72:076901. doi:10.1088/00344885/72/7/076901

Acernese F, Agathos M, Agatsuma K et al (2015) Advanced Virgo: a second-generation interferometric gravitational wave detector. Class Quantum Grav 32:024001. doi:10.1088/0264-9381/32/2/ 024001

Akutsu T (2015) Large-scale cryogenic gravitational-wave telescope in Japan: KAGRA (for the KAGRA collaboration). J Phys 610:012016. doi:10.1088/1742-6596/610/1/012016

Dolgikh G, Ovcharenko V (2008) Earth's noise in the range of infragravity sea waves. Dokl Earth Sci 422:1129-1132

Dolgikh G, Valentin D, Dolgikh S, Kovalev S, Koren' I, Ovcharenko V, Fishchenko V (2002) Application of horizontally and vertically oriented strainmeters in geophysical studies of transitional zones. Izv Phys Solid Earth 38:686-689

Dolgikh G, Dolgikh S, Kovalev S, Koren I, Novikova O, Ovcharenko V, Okuntseva O, Shvets V, Chupin V, Yakovenko S (2004) A laser nanobarograph and its application to the study of pressurestrain coupling. Izv Phys Solid Earth 40(8):683-691

Dolgikh G, Dolgikh S, Kovalev S, Chupin V, Shvets V, Yakovenko S (2007) A deformation method for determining the tsunami potential of earthquakes. Dokl Earth Sci 417:1261 
Garoi F, Apostol D, Damian V, Logofatu P, Ionita B, Lazar J, Molesini G, Papadopoulos T, Ionescu C, Tugui A (2008) Proceedings of SPIE 7022702218

Jahr T, Kroner C, Lippmann A (2006) Strainmeters at Moxa observatory, Germany. J Geodyn 41:205

McDonnell M, Stock N, Pearce C, Abbott D (2008) Stochastic resonance: from suprathreshold stochastic resonance to stochastic signal quantization. Cambridge University Press, Cambridge

Park J, Amoruso A, Crescentini L, Boschi E (2008) Long-period toroidal Earth free oscillations from the great Sumatra-Andaman earthquake observed by paired laser extensometers in Gran Sasso, Italy. Geophys J Int 173:887

Takemoto S, Araya A, Akamatsu J, Morii W, Momose H, Ohashi M, Kawasaki I, Higashi T, Fukuda Y, Miyoki S, Uchiyama T, Tatsumi D, Hanada H, Naito I, Telada S, Ichikawa N, Onoue K, Wada Y (2004) A 100 m laser strainmeter system installed in a $1 \mathrm{~km}$ deep tunnel at Kamioka, Gifu, Japan. J Geodyn $38: 477-488$ 